\title{
Article
}

\section{Follow-Up of Extended Shells around B[e] Stars}

\author{
Tiina Liimets $^{1, *(\mathbb{D})}$, Michaela Kraus ${ }^{1} \mathbb{D}$, Alexei Moiseev ${ }^{2}$, Nicolas Duronea ${ }^{3}$, Lydia Sonia Cidale ${ }^{3,4}$ \\ and Cecilia Fariña ${ }^{5,6}$
}

1 Astronomical Institute, Czech Academy of Sciences, Fričova 298, 25165 Ondrejov, Czech Republic; michaela.kraus@asu.cas.cz

2 Special Astrophysical Observatory, Russian Academy of Sciences, 369167 Nizhnii Arkhyz, Russia; moisav@gmail.com

3 Instituto de Astrofísica de La Plata (CCT La Plata-CONICET, UNLP), Paseo del Bosque S/N, La Plata B1900FWA, Buenos Aires, Argentina; nduronea@fcaglp.unlp.edu.ar (N.D.); lydia@fcaglp.unlp.edu.ar (L.S.C.)

4 Departamento de Espectroscopía, Facultad de Ciencias Astronómicas y Geofísicas, Universidad Nacional de La Plata, Paseo del Bosque S/N, La Plata B1900FWA, Buenos Aires, Argentina

5 Isaac Newton Group, Apartado de Correos 321, 38700 Santa Cruz de La Palma, Canary Islands, Spain; cf@ing.iac.es

6 Instituto de Astrofísica de Canarias, 38205 La Laguna, Tenerife, Spain

* Correspondence: tiina.liimets@asu.cas.cz

check for updates

Citation: Liimets, T.; Kraus, M.; Moiseev, A.; Duronea, N.; Cidale, L.S.; Fariña, C. Follow-Up of Extended Shells around B[e] Stars. Galaxies 2022, 10, 41. https:/ / doi.org/10.3390/galaxies10020041

Academic Editors: Martín Guerrero, Noam Soker and Quentin A. Parker

Received: 30 January 2022

Accepted: 25 February 2022

Published: 1 March 2022

Publisher's Note: MDPI stays neutral with regard to jurisdictional claims in published maps and institutional affiliations.

Copyright: (c) 2022 by the authors. Licensee MDPI, Basel, Switzerland. This article is an open access article distributed under the terms and conditions of the Creative Commons Attribution (CC BY) license (https:/ / creativecommons.org/licenses/by/ $4.0 /)$.

\begin{abstract}
B}[\mathrm{e}]$ stars are massive B type emission line stars in different evolutionary stages ranging from pre-main sequence to post-main sequence. Due to their mass loss and ejection events these objects deposit huge amounts of mass and energy into their environment and enrich it with chemically processed material, contributing significantly to the chemical and dynamical evolution of their host galaxies. However, the large-scale environments of these enigmatic objects have not attracted much attention. The first and so far only catalog reporting the detection of extended shells around a sample of $\mathrm{B}[\mathrm{e}]$ stars was an $\mathrm{H} \alpha$ imaging survey carried out in the year 2001, and was limited to bright targets in the northern hemisphere. We have recently started a follow-up of those targets to detect possible evolution of their nebulae in the plane of the sky over a baseline of two decades. Furthermore, we extend our survey to southern targets and fainter northern ones to complement and complete our knowledge on large-scale ejecta surrounding B[e] stars. Besides imaging in $\mathrm{H} \alpha$ and selected nebular lines, we utilize long-slit and 3D spectral observations across the nebulae to derive their physical properties. We discovered pronounced nebula structures around 15 more objects, resulting in a total of $27 \mathrm{~B}[\mathrm{e}]$ stars with a large-scale nebula. Here we present our (preliminary) results for three selected objects: the two massive supergiants MWC137 and MWC 314, and the unclassified B[e] star MWC 819.
\end{abstract}

Keywords: circumstellar matter; stars: individual (MWC 137, MWC 314, MWC 819); stars: massivesupergiants

\section{Introduction}

$\mathrm{B}[\mathrm{e}]$ stars are massive B-type emission-line stars. Their spectra exhibit intense Balmer emission, numerous forbidden lines, as well as permitted lines of low ionized metals. These stars can be found in different evolutionary stages, from pre- to post-main sequence and spreading over a large mass range (from sub-solar up to $70 M_{\odot}$ for the currently known members ${ }^{1}$ ). In addition, their spectral energy distributions display a strong infrared excess due to the hot, 500 to $1000 \mathrm{~K}$, circumstellar dust (e.g., [1]). This dust is suggested to be located in a ring or disk-like structure based on polarimetric observations. The confinement of the dust into a disk has been confirmed by interferometric observations of the closest and brightest objects [2-4]. Furthermore, detailed analysis of interferometric and highresolution spectroscopic observations revealed that the stars are surrounded by multiple rings of gas that revolve the central object on (quasi-)Keplerian orbits (see, e.g., [3-7]). The location of the dust can (but must not) coincide with some individual gas rings. The 
distribution of rings around each object is found to be unique and independent on whether the star is presumably single or part of a binary system [6]. The composition and dynamics of these small-scale discs (several AU), in particular around the B[e] supergiants, have been studied intensively to unveil their possible formation mechanisms for which critical rotation, binary interaction, as well as slow-wind solutions and pulsation triggered ejections are among the suggested scenarios (for details see reviews [8,9]).

In addition to the small-scale dusty discs, B[e] stars are surrounded by large-scale (up to several pc) ionized gas, which has not been studied much. A pioneering work [10] presented an $\mathrm{H} \alpha$ imaging survey for 25 northern $\mathrm{B}[\mathrm{e}]$ stars, and report the detection of extended shells around 50\% of the targets with various shapes: spherical and ring nebulae, spiral-arm like features, bipolar and unipolar-lobe structures. This diversity in shapes indicates that the formation mechanism is most likely not a unique process. Furthermore, the physical mechanism creating such mass ejection is not well known. Therefore, to have a statistically meaningful sample of $\mathrm{B}[\mathrm{e}]$ targets with extended nebulae, and to investigate details of their mass-loss history we started an observational campaign with two goals. Firstly, to perform a follow-up of the targets with already detected nebulae. Secondly, to enlarge the sample of $\mathrm{B}[\mathrm{e}]$ stars with confirmed nebulae by adding fainter, yet unexplored objects located in the north and by extending the survey to the southern sky. In this proceedings we present results for selected objects of our project.

\section{Observations and Data Reduction}

Our follow-up $\mathrm{H} \alpha$ imaging survey is mostly carried out with the Nordic Optical Telescope $^{2}$ (NOT) at La Palma and with the Danish Telescope in La Silla, for the northern and southern targets, respectively. Average exposure times are 15-30 min. For deeper images of selected targets GMOS [11,12] at Gemini South was used while for very large nebulae we utilized the Isaac Newton Telescope (INT). Complementing long-slit spectroscopic observations have been secured already for a small sample of objects. These observations extend over the wavelength range from 6350 to $6850 \AA$ with a spectral resolution of $\mathrm{R} \sim 10,000$ and have been carried out at the NOT as well. This wavelength range covers nebular emission lines-H $\alpha 6563 \AA$, [NII] $\lambda \lambda 6548,6583$, [SII] $\lambda \lambda$ 6716,6731. In addition, we have exploited the Scanning Fabry-Perot Interferometer (FPI) mounted at the Russian $6 \mathrm{~m}$ Big Telescope Alt-azimuth (BTA). More observational details of our survey will be presented in a forthcoming paper (Liimets et al. in prep). In this proceedings we will restrict to details about observations and data reduction for the targets under discussion. These are the two supergiant stars MWC 137, and MWC 314, and the object MWC 819, which currently has an unknown evolutionary state.

The $\mathrm{H} \alpha$ images of the B[e] supergiant MWC 137 were obtained with the NOT and the Danish Telescope in 2016 and 2019, respectively. For the spectral observations the long-slit possiblities at the NOT as well as the scanning FPI mode at the BTA were exploited. Further observational details of the data used to investigate MWC 137 can be found from [13,14].

The imaging observations of the large-scale bipolar nebula of MWC 314 have been performed with the Wide Field Camera at the INT, which provides a Field of View (FOV) $33^{\prime} \times 33^{\prime}$ and a pixel scale $0^{\prime \prime} .33 \mathrm{pix}^{-1}$. On 3 October 2019 five dithered frames with an exposure time of $10 \mathrm{~min}$ each were acquired totalling $50 \mathrm{~min}$. The narrowband $\mathrm{H} \alpha$ filter No. 196 was used, which includes also the [NII] doublet. Standard routines in IRAF $^{3}$ were used for the data reduction. Spectral observations of MWC 314 were performed at the BTA with the multi-mode focal reducer SCORPIO-2 [15], which provides FOV $6^{\prime} \times 6^{\prime}$ with a $0^{\prime \prime} .4$ pixel scale in the $2 \times 2$ binning mode. To cover the entire nebula, it had to be split into four fields. The 3D spectroscopic cubes obtained in the scanning FPI mode have a resolution of $\mathrm{R} \sim 16,000$ in the narrow spectral range containing the $\mathrm{H} \alpha$ emission line. The observed four fields were merged into a mosaic cube with an angular size $18^{\prime} \times 12^{\prime}$. Details of the FPI data reduction can be found in [16].

A new deep $\mathrm{H} \alpha$ image of MWC 819 was acquired on 15 November 2020 with GMOS attached to Gemini South (program ID GS-2020B-Q-210). The FOV was $5^{\prime} .5 \times 5^{\prime} .5$ with a pixel 
scale $0^{\prime \prime} .16 \mathrm{pix}^{-1}$ in the utilized $2 \times 2$ binning mode. The observations were taken with filter G0336, which covers, besides $\mathrm{H} \alpha$, also the nebular lines from the [NII] $\lambda \lambda 6548,6583$ doublet. To remove the gaps between the CCDs the total exposure time of $10 \mathrm{~min}$ was divided into 5 subframes of 2 min each. For the data reduction the official Gemini Observatory software DRAGONS [17] was used.

Finally, we have at our hands the raw $\mathrm{H} \alpha$ images from 2001 observed with the Mt. Palomar 60 inch telescope. Details about the observations are given in [10]. We have reduced the images using standard IRAF routines.

\section{Results and Discussion}

Our $\mathrm{H} \alpha$ imaging survey has been completed. To the already published $\mathrm{H} \alpha$ survey of $25 \mathrm{~B}[\mathrm{e}]$ stars [10], we added 32 objects, 9 in the north and 23 in the southern sky. Similarly to the first survey, we report detecting large-scale nebular features around $50 \%$ of the observed stars, that is 4 in the north and 11 in the south. The full description of our survey will be published separately (Liimets et al. in prep), but the results for five objects from our sample (MWC 137, [GKF2010] MN 83, [GKF2010] MN 108, [GKF2010] MN 109, and [GKF2010] MN 112) have been presented in $[13,14,18]$. In the following, we summarize our work on MWC 137 and present preliminary results for two additional objects: MWC 314 and MWC 819.

\subsection{MWC 137}

The object MWC 137 has been classified as a supergiant star that has just left the main sequence. Its mass and age have been estimated to be $37_{-5}^{+9} M_{\odot}$ and $4.7 \pm 0.8 \mathrm{Myr}$, respectively [14]. Our detailed investigations of the various data sets presented in $[13,14]$ have led to the following picture of the environs of the B[e] supergiant MWC 137.

The central star is surrounded by hot $\mathrm{CO}$ gas forming a subarcsecond size disc displaying Keplerian rotation. Further out from the center a prominent arcminute scale ionized nebula is visible (Figure 1). In the Figure, the emission from the $\mathrm{H} \alpha+[\mathrm{NII}]$ doublet is depicted in blue, while with red we present the warm and cool dust observed with the Spitzer Space Telescope in the $3.6 \mu \mathrm{m}$ band. The dust visible in the infrared has a slightly larger size than the optical nebula, appart from the north-west (NW) direction, where the warm and cool dust seems to be absent (see Figure 1). The ionized gas has an elliptical shape with varying intensity areas, presenting several brigther filaments. We compared our newly obtained $\mathrm{H} \alpha$ image from 2019 with the image from 2001 [10]. Our baseline of 18.1 years did not reveal any morphological changes. Neither could we yet detect any expansion in the plane of the sky.

We further investigated the ionzied gas in the optical range using our various spectral observations. In Figure 2 left we present our radial velocity (RV) measurements of the combined [SII] $\lambda \lambda 6716,6731$ doublet obtained with the scanning FPI which is providing spectral coverage over the whole FOV. The RV pattern is complex, which is also supported with our long-slit spectra obtained with the NOT+ALFOSC ${ }^{4}$ at various position angles across the nebula (see Figure 4 in [13] and Figure 5 in [14]). However, the northern part is mostly blue shifted and the southern part red shifted. In addition, from our long-slit spectra we could estimate electron densities utilizing the same [SII] doublet and assuming a nebular temperature of 10,000 K. Similarly to the RV, the electron densities show a varying pattern across the nebula, from 0 to $800 \mathrm{~cm}^{-3}$ (Figure 2 right). The higher densities are at the regions of intense emission and at the central area. 


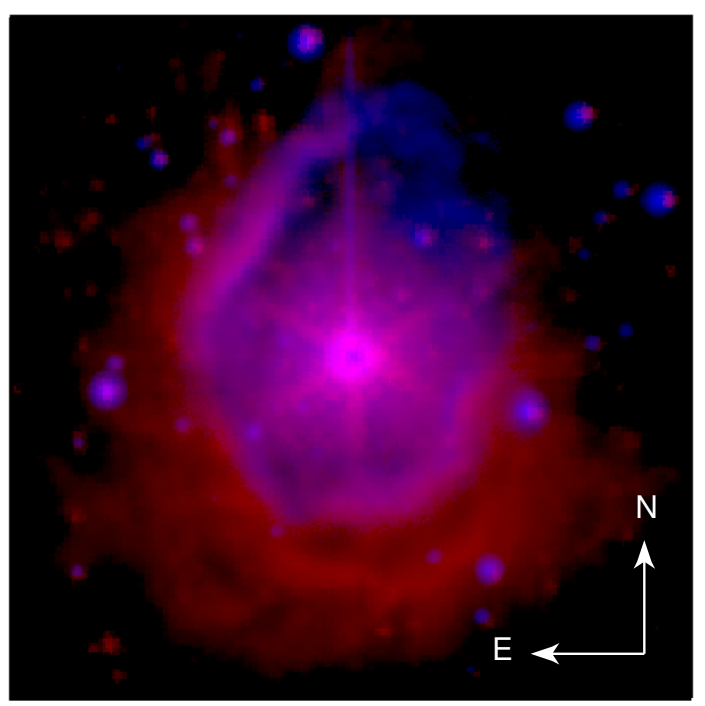

Figure 1. Large-scale nebula of MWC 137. ALFOSC $\mathrm{H} \alpha$ iamge with blue and the Sptizer $3.6 \mu \mathrm{m}$ with red. FOV $2^{\prime} \times 2^{\prime}$. Figure taken from [13] (their Figure 10, (c) AAS, reproduced with permission).
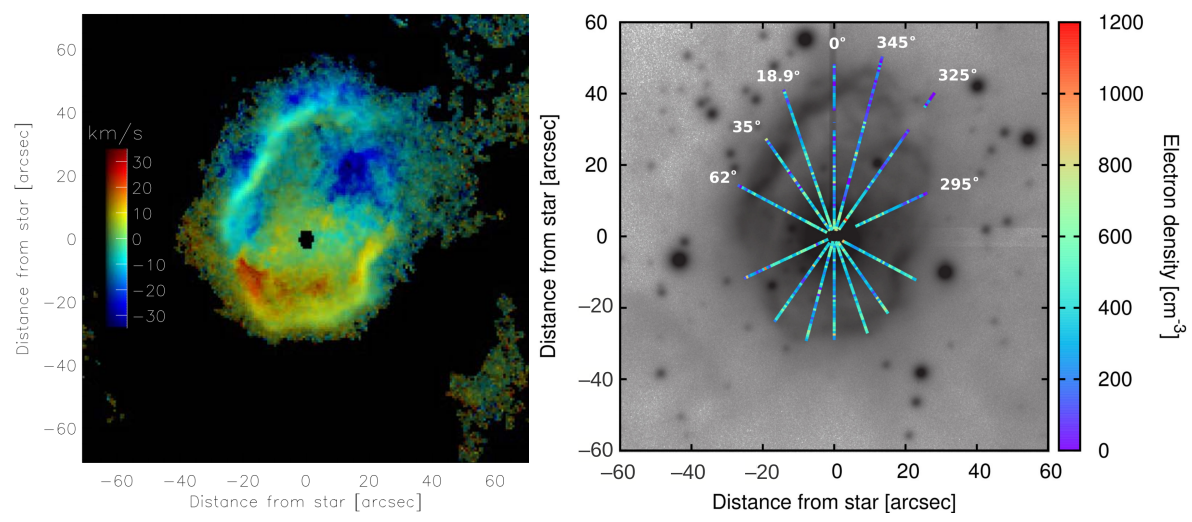

Figure 2. Radial velocity measurements of the MWC 137 from the FPI observations (left) and electron density distribution across the nebula obtained from the ratio of the [SII] $\lambda \lambda 6716,6731$ lines measured in the NOT+ALFOSC long-slit spectra (right). On both panels north is up and east to the left. Figures taken from [14] (their Figure 4a, left panel, and Figure 9, right panel, (C) AAS, reproduced with permission).

Moving to even larger distances from the center of MWC 137 we find that the optical nebula is surrounded by a vast amount of cold molecular gas (see Figure 12 in [13]) detected with the Atacama Pathfinder EXperiment (APEX). The various molecular clouds resolved in the radio regime have masses from 15 to $245 M_{\odot}$ and seem to embrace the ionized nebula in the west, south, and east, clearly implying an interstellar origin rather than resulting from the evolution of the central star.

\subsection{MWC 314}

MWC 314 is one of the most luminous stars in the Galaxy [19]. The debate over its nature is ongoing-a Luminous Blue Variable (LBV, e.g., [20]) or a B[e] supergiant (e.g., [21]). It seems to be established though that the central object is a contact binary. But the masses of the system and of the individual stars are also controversial. Suggested are a total mass of the system of $66 \pm 9 M_{\odot}$ [22] and of $\sim 20 M_{\odot}$ [23]. This discrepancy renders it difficult to assign the object a proper evolutionary state. MWC 314 is surrounded by a huge bipolar nebula extending $18^{\prime}$ across ([10], Figure 3), which considering the distance of $3 \mathrm{kpc}$ [19] translates into a physical size larger than $13 \mathrm{pc}$. 
We started the analysis with our $\mathrm{H} \alpha$ image from 2019, which we compared to the one from 2001 to find any morphological changes and/or expansion in the plane of the sky. Comparing the bipolar lobes in the east-west direction, no morphological changes were detected. For the expansion in the plane of the sky, we exploited the so-called magnification method (see e.g., [24,25]), which is able to detect expansion down to 0.1 pixels on the residual image that has been created from the images taken at two different dates. To properly apply the magnification method, further processing of the images was needed, which included pixel by pixel, seeing, and flux matching. Further details of this processing can be found from [14,25]. Unfortunately, our baseline of 18 years did not reveal any expansion in the plane of the sky.

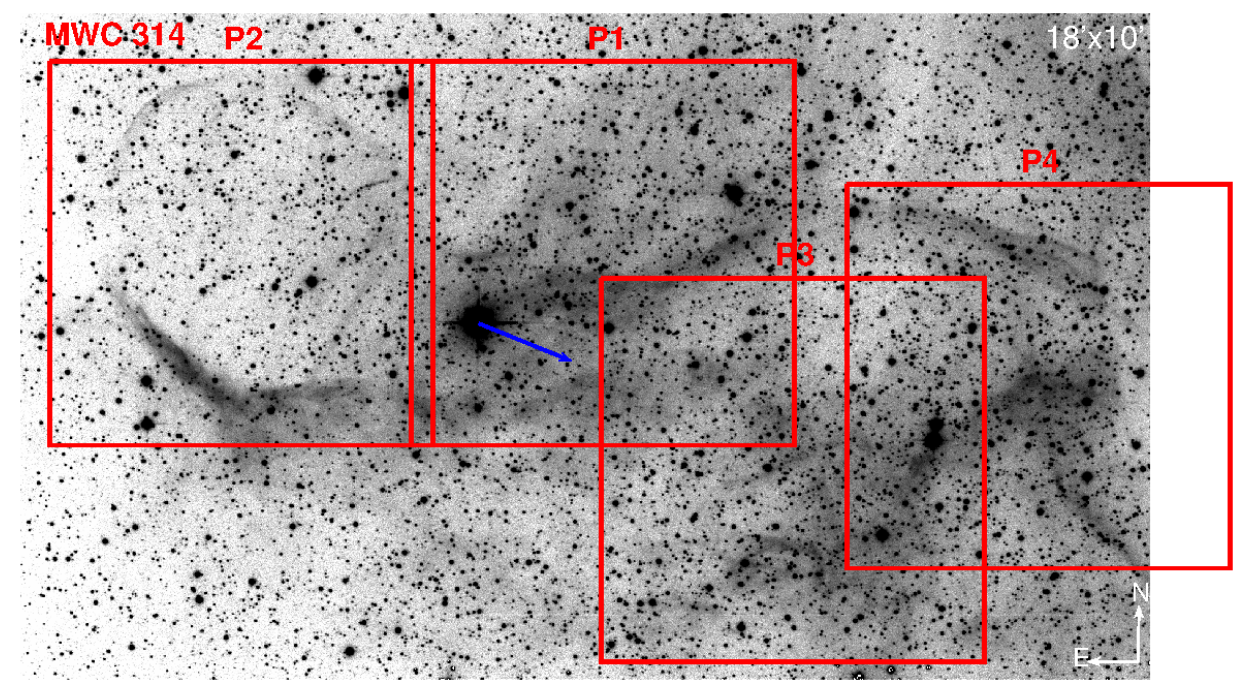

Figure 3. $\mathrm{H} \alpha$ image (INT) of the bipolar nebula around MWC 314. The pointings for the spectroscopic FPI observations are shown in red. See text for more details.

Next, from inspection of our deep image, a possible third lobe NW of the central object might be identified, which is barely seen on the image from 2001 [10]. To further investigate this lobe, we included it into our spectroscopic observations with the FPI. It is almost fully covered by the pointing P1 (see Figure 3). The other pointings were chosen such that they cover most of the north-east (NE) lobe (P2) and the lobe in the west (W, P3 and P4). The proper motion of the central star, according to Gaia DR2 [26], is -2.3 mas year ${ }^{-1}$ and -4.8 mas year $^{-1}$ in Right Ascension and Declination, respectively. A blue arrow, indicating the direction of this movement, is drawn in Figure 3, which fits well with a plausible swept-back nature of the bipolar nebula similar to the swept-back nebula detected around the classical nova GK Persei 1901 (see Figure 2 in [27]). This could also mean that the western lobe, which has been previously considered in the literature as the plausible counterpart to the NE lobe, must not necessarily be connected to MWC 314. In fact, the NE lobe appears to be rather extended towards the west and south, but these features have no clear counterparts in NE direction.

To test whether the NW structure is the actual counterpart to the NE lobe, velocity measurements over the entire nebula are required. Unfortunately, the 3D spectroscopic observation (see Figure 4) had no signal in that area. However, as pointed out by Bruce Balick during the conference, our proposed new lobe in the NW seems to be replenished with gas, while the two previously considered lobes are not. This is, so far, the only argument speaking against the scenario of the new lobe to be related to the MWC 314 . Without further observations, in particular a repetition of the velocity measurements, we cannot confirm yet the possible connection of the NW lobe with MWC 314. 


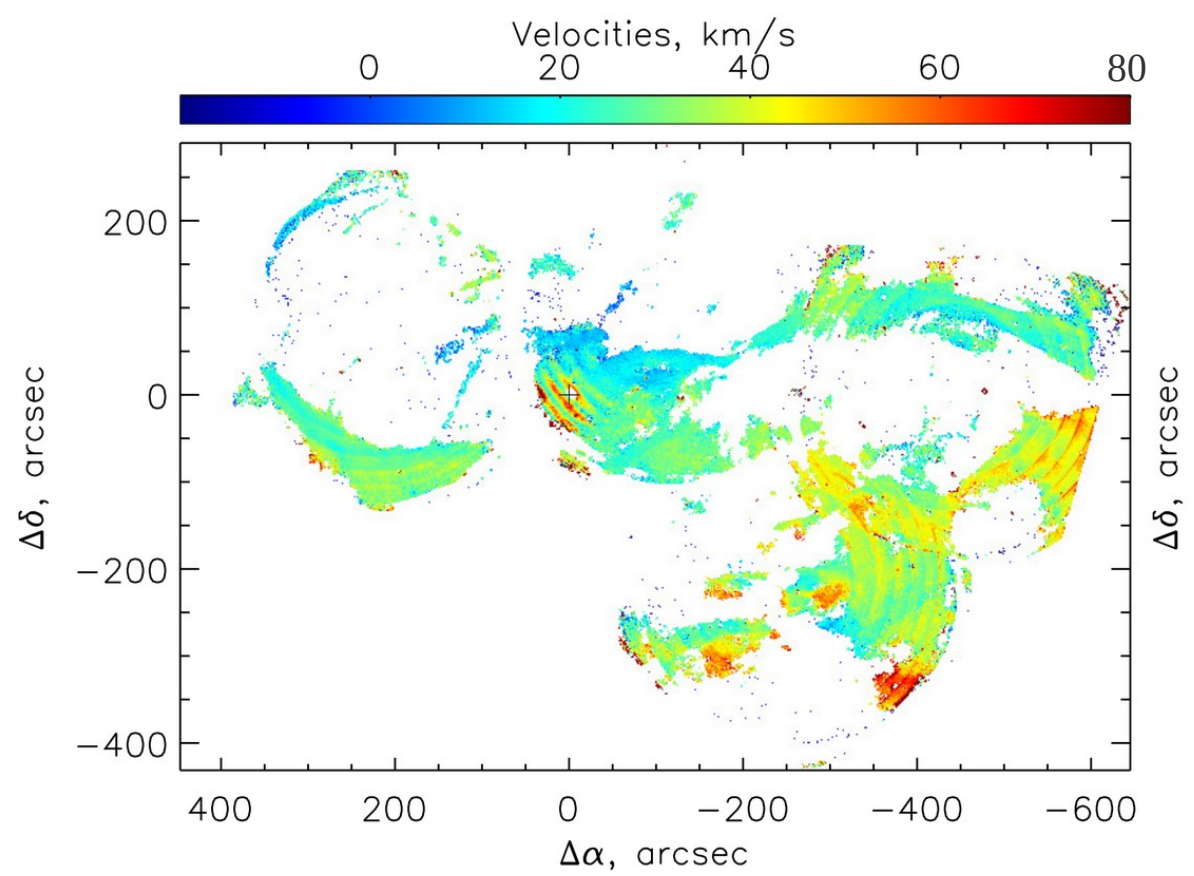

Figure 4. Velocity field of the bipolar nebula around MWC 314 measured from the [SII] lines observed with the FPI.

Thirdly, we analysed the velocity field of the lobes of MWC 314 which is presented in Figure 4. As was already mentioned, the signal-to-noise was too low to get any measurements at the position of the NW lobe. In addition, due to problems with subtraction of the bright airglow emission lines, the RV measurements suffer from artefacts, which appear in Figure 4 as concentric rings with artificially higher radial velocities. Further analysis using new data reduction algorithms is required to remove these spurious signals. Nevertheless, from the RV figure we can tentatively conclude that the northern parts are predominantly blue shifted while the southern parts are redder, broadly from $-15 \mathrm{~km} \mathrm{~s}^{-1}$ to $+40 \mathrm{~km} \mathrm{~s}^{-1}$, respectively.

\subsection{MWC 819}

MWC 819 has long been listed as an unclassified B[e] star, but recently, [28] concluded from their analysis of new optical and infrared data that it might be a compact protoplanetary nebula. Consequently, the central object should currently be evolving into a white dwarf. On the left side panel of Figure 5 we present the image from 2001 [10] and on the right side panel we show our $\mathrm{H} \alpha$ image from 2020. The FOV of each image is $3^{\prime} .6 \times 3^{\prime} .1$ and MWC 819 is the bright source in the center of each image.

From the 2001 data MWC 819 was identified as having a unipolar nebula [10], visible as the faint roundish nebular feature in the NE. Our new and considerably deeper image resolves the previously detected features in more detail and reveals an additional possibly related nebular ejecta. In particular, the "unipolar lobe" (marked with a big black circle in our 2020 image) for which a zoom-in is presented with an orange square (FOV $38^{\prime \prime} \times 36^{\prime \prime}$ ), resembles an individual nebula around a faint, possibly background object that was not detected in the 2001 image. To confirm this hypothesis, additional observations and analyses are needed. We would also like to draw the readers attention to the smaller nebular features in the south-west (SW) marked with two smaller black circles. Those features were not mentioned in [10]. However, after being clearly resolved in our deeper image, faint counterparts can be detected also in the 2001 image. Furthermore, with red we mark previously not detected features. One in the NE and the other in the NW direction. The latter is especially interesting, because due to its position perpendicular to the main nebular features in the NE to SW direction, it might be possible that a jet is emanating from 
MWC 819. In fact, jets have been discovered in a few other B[e] stars (e.g., MWC 137 [13,29], MWC 922 [30], Z CMa [31]).

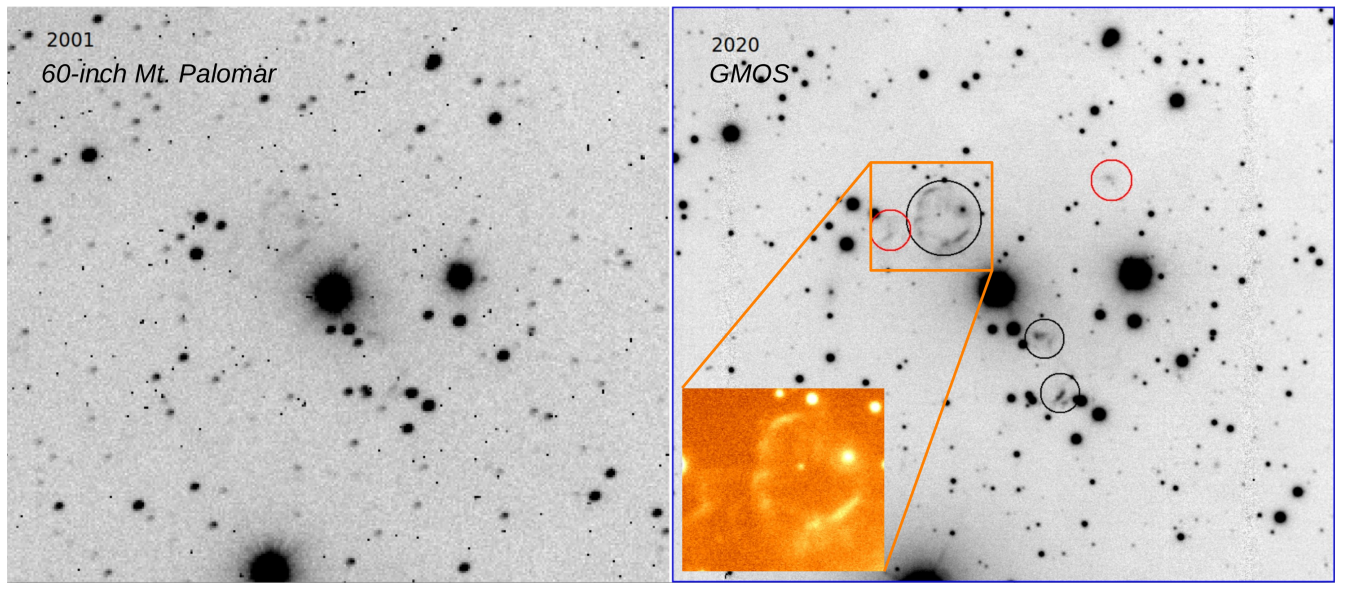

Figure 5. Nebular features of the B[e] star MWC 819 seen in 2001 from [10] (left) and in our new, deeper image taken in 2020 (right). North is up, east is left.

\section{Conclusions}

We have performed a new $\mathrm{H} \alpha$ imaging survey of $32 \mathrm{~B}[\mathrm{e}]$ stars. Of these, 9 targets are located in the northern hemisphere, and 23 in the southern one. Moreover, we re-observed the $25 \mathrm{~B}$ [e] stars, whose environment had been imaged about two decades ago [10]. Our study has two main goals: (i) to search for indications of large-scale ejecta around the yet unstudied objects, and (ii) to investigate the morphology of the nebulae and their expansion dynamics. Furthermore, all objects with detected circumstellar nebulae are observed spectroscopically in a systematic way to derive the physical parameters such as temperature and density distribution across the nebulae together with their ionization structure and chemical composition. The combined information will be used to address the issue of the yet unknown mass-loss history of these enigmatic objects. Moreover, we expect that the detailed information about the nebulae gained during our project will help to settle the yet unknown evolutionary state of many of the objects.

Our survey of the newly imaged objects has revealed that about $50 \%$ (15 objects out of 32) of the $B[e]$ stars have detectable nebular features. This detection rate is similar to the previously reported one (12 out of 25 objects, [10]). The shapes of nebulae display a high diversity, and a full catalog with all the images is in preparation.

While for most objects the analysis is still ongoing and, in particular, a wealth of spectroscopic data still needs to be acquired, we have presented our (preliminary) results for three objects. Two of them (MWC 137 and MWC 314) had been previously imaged, but our current baseline of about 18 years provided neither evidence for changes in nebular morphology nor indications for nebular expansions. However, both objects have rather large distances (about 5 and $3 \mathrm{kpc}$, respectively), so that our current spatial resolution is still too coarse to expect detectable changes, given the moderate radial velocities measured in both nebulae. Our deep image of the presumably bipolar nebula around MWC 314 pointed to a third, faint lobe filled with emission, which would fit better to the direction of the proper motion of the star. Whether it can indeed be associated with MWC 314 requires better radial velocity measurements of the entire nebular structure than the one we could secure so far.

For the third object presented, MWC 819, we have also an old set of recorded data at hand. Our new, significantly deeper image suggests that one of the previously detected nebular features has been mistakenly identified as uni-polar lobe connected to MWC 819 . It seems that this feature could be associated instead to a faint back-ground source, which was not visible on the old image. However, our new, high-quality image displays other, yet unreported signs for ejected matter, maybe in relation with a jet. Confirmation is still 
pending and requires high-quality spectra, which are currently under way, to determine the velocity structure of the detected features.

Author Contributions: Conceptualization and Project administration, T.L., M.K. and L.S.C.; Resources and Data curation: T.L., A.M., N.D., L.S.C. and C.F.; Formal analysis, Investigation, Software, Methodology and Validation, T.L., M.K., A.M. and N.D.; Supervision, M.K.; Visualization, T.L., M.K. and A.M.; writing—original draft preparation, T.L. and M.K.; writing—review and editing, all; Funding acquisition, M.K. and L.S.C. All authors have read and agreed to the published version of the manuscript.

Funding: This research was funded by the Czech Science foundation (GA ČR, grant number 2000150S), by CONICET (PIP 0177, PIP 2893) and by the Agencia Nacional de Promoción Científica y Tecnológica (PICT 2016-1971). The Astronomical Institute of the Czech Academy of Sciences is supported by the project RVO:67985815. This project has also received funding from the European Union's Framework Programme for Research and Innovation Horizon 2020 (2014-2020) under the Marie Skłodowska-Curie Grant Agreement No. 823734. The analysis of SCORPIO-2 data was carried out within the framework of the government contract of SAO RAS approved by the Ministry of Science and Higher Education of the Russian Federation.

Institutional Review Board Statement: Not applicable.

Informed Consent Statement: Not applicable.

Data Availability Statement: Most of the data presented in these proceedings can be acquired using the following public archives: NOT FITS Header Archive ${ }^{5}$ for NOT data, Isaac Newton Group Archive $^{6}$ for INT data, Gemini Observatory Archive ${ }^{7}$ for GMOS data, ESO Archive ${ }^{8}$ for APEX data, and The General Observation Archive ${ }^{9}$ for BTA data. Observations obtained with the Danish and Mt. Palomar 60 inch telescope can be obtained from TL on a reasonable request.

Acknowledgments: We thank the anonymous referees for their constructive comments that helped to improve the manuscript. This research made use of the NASA Astrophysics Data System (ADS) and of the SIMBAD database, operated at CDS, Strasbourg, France. This publication is based on observations obtained at the international Gemini Observatory, a program of NSF's NOIRLab [processed using DRAGONS (Data Reduction for Astronomy from Gemini Observatory North and South)], which is managed by the Association of Universities for Research in Astronomy (AURA) under a cooperative agreement with the National Science Foundation on behalf of the Gemini Observatory partnership: the National Science Foundation (United States), National Research Council (Canada), Agencia Nacional de Investigación y Desarrollo (Chile), Ministerio de Ciencia, Tecnología e Innovación (Argentina), Ministério da Ciência, Tecnologia, Inovações e Comunicações (Brazil), and Korea Astronomy and Space Science Institute (Republic of Korea) under program ID GS-2020B-Q-210. Partially based on observations made with the Nordic Optical Telescope, owned in collaboration by the University of Turku and Aarhus University, and operated jointly by Aarhus University, the University of Turku and the University of Oslo, representing Denmark, Finland and Norway, the University of Iceland and Stockholm University at the Observatorio del Roque de los Muchachos, La Palma, Spain, of the Instituto de Astrofisica de Canarias. The data presented here were obtained in part with ALFOSC, which is provided by the Instituto de Astrofisica de Andalucia (IAA) under a joint agreement with the University of Copenhagen and NOT. Observations on the 6-m telescope of SAO RAS are supported by the Ministry of Science and Higher Education of the Russian Federation (including contract No. 05.619.21.0016, unique project identifier RFMEFI61919X0016). The Isaac Newton Telescope is operated on the island of La Palma by the Isaac Newton Group of Telescopes in the Spanish Observatorio del Roque de los Muchachos of the Instituto de Astrofísica de Canarias. We thank Bruce Balick for a valuable discussions on the nebula of MWC 314

Conflicts of Interest: The authors declare no conflict of interest. 


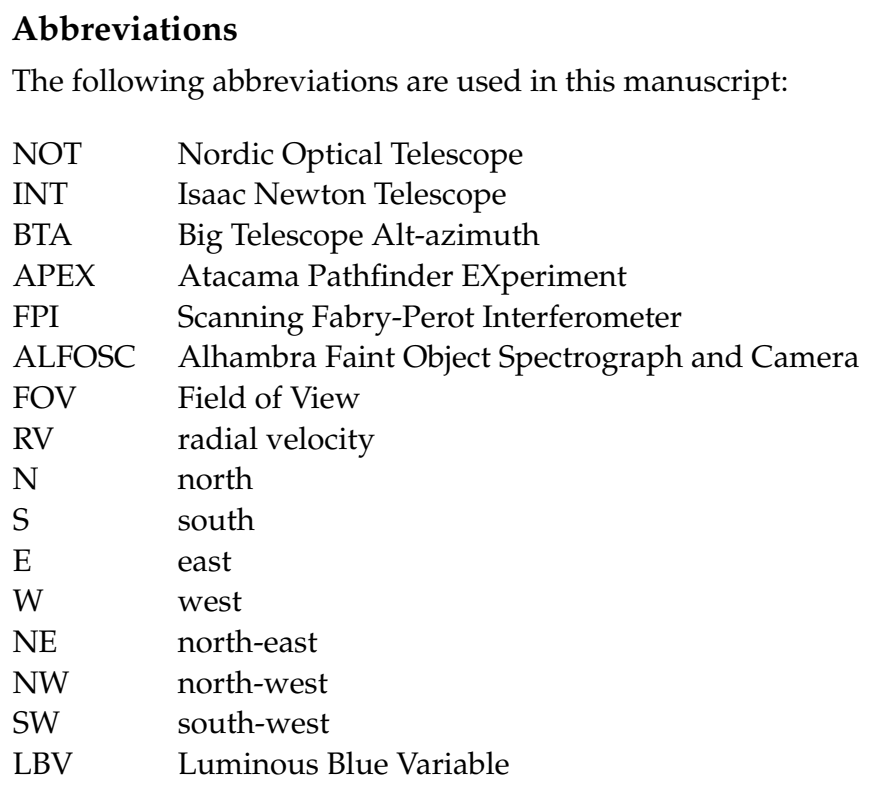

\section{Notes}

1 We would like to emphasize that stars with the $\mathrm{B}[\mathrm{e}]$ phenomenon are not predicted by any of the currently known stellar evolution theories. This might be related to the fact that these models are purely $1 \mathrm{D}$ and do not account for aspherical mass loss, which is typically seen in $\mathrm{B}[\mathrm{e}]$ stars. Hence this limit of $70 \mathrm{M}_{\odot}$ might not be real and more massive B[e] stars might exist, which just have not been discovered yet.

2 http://www.not.iac.es/ (accessed on 31 January 2022).

3 IRAF is distributed by the National Optical Astronomy Observatory, which is operated by the Association of Universities for Research in Astronomy (AURA) under cooperative agreement with the National Science Foundation.

\section{References}

1. Lamers, H.J.G.L.M.; Zickgraf, F.J.; de Winter, D.; Houziaux, L.; Zorec, J. An improved classification of B[e]-type stars. Astron. Astrophys. 1998, 340, 117-128.

2. Domiciano de Souza, A.; Driebe, T.; Chesneau, O.; Hofmann, K.H.; Kraus, S.; Miroshnichenko, A.S.; Ohnaka, K.; Petrov, R.G.; Preisbisch, T.; Stee, P.; et al. AMBER/VLTI and MIDI/VLTI spectro-interferometric observations of the B[e] supergiant $<$ ASTROBJ $>$ CPD $-57^{\circ} 2874</$ ASTROBJ $>$. Size and geometry of the circumstellar envelope in the near- and mid-IR. Astron. Astrophys. 2007, 464, 81-86. [CrossRef]

3. Millour, F.; Meilland, A.; Chesneau, O.; Stee, P.; Kanaan, S.; Petrov, R.; Mourard, D.; Kraus, S. Imaging the spinning gas and dust in the disc around the supergiant A[e] star HD 62623. Astron. Astrophys. 2011, 526, A107, [CrossRef]

4. Cidale, L.S.; Borges Fernandes, M.; Andruchow, I.; Arias, M.L.; Kraus, M.; Chesneau, O.; Kanaan, S.; Curé, M.; de Wit, W.J.; Muratore, M.F. Observational constraints for the circumstellar disk of the B[e] star CPD-52 9243. Astron. Astrophys. 2012, 548, A72. [CrossRef]

5. $\quad$ Kraus, M.; Cidale, L.S.; Arias, M.L.; Maravelias, G.; Nickeler, D.H.; Torres, A.F.; Borges Fernandes, M.; Aret, A.; Curé, M.; Vallverdú, R.; et al. Inhomogeneous molecular ring around the B[e] supergiant LHA 120-S 73. Astron. Astrophys. 2016, 593, A112, [CrossRef]

6. Maravelias, G.; Kraus, M.; Cidale, L.S.; Borges Fernandes, M.; Arias, M.L.; Curé, M.; Vasilopoulos, G. Resolving the kinematics of the discs around Galactic B[e] supergiants. Mon. Not. R. Astron. Soc. 2018, 480, 320-344. [CrossRef]

7. Torres, A.F.; Cidale, L.S.; Kraus, M.; Arias, M.L.; Barbá, R.H.; Maravelias, G.; Borges Fernandes, M. Resolving the clumpy circumstellar environment of the B[e] supergiant LHA 120-S 35. Astron. Astrophys. 2018, 612, A113, [CrossRef]

8. de Wit, W.J.; Oudmaijer, R.D.; Vink, J.S. Dusty Blue Supergiants: News from High-Angular Resolution Observations. Adv. Astron. 2014, 2014, 270848, [CrossRef] 
9. Kraus, M. A Census of B[e] Supergiants. Galaxies 2019, 7, 83, [CrossRef]

10. Marston, A.P.; McCollum, B. Extended shells around B[e] stars. Implications for B[e] star evolution. Astron. Astrophys. 2008, 477, 193-202. [CrossRef]

11. Hook, I.M.; Jørgensen, I.; Allington-Smith, J.R.; Davies, R.L.; Metcalfe, N.; Murowinski, R.G.; Crampton, D. The Gemini-North Multi-Object Spectrograph: Performance in Imaging, Long-Slit, and Multi-Object Spectroscopic Modes. Publ. Astron. Soc. Pac. 2004, 116, 425-440. [CrossRef]

12. Allington-Smith, J.; Murray, G.; Content, R.; Dodsworth, G.; Davies, R.; Miller, B.W.; Jorgensen, I.; Hook, I.; Crampton, D.; Murowinski, R. Integral Field Spectroscopy with the Gemini Multiobject Spectrograph. I. Design, Construction, and Testing. Publ. Astron. Soc. Pac. 2002, 114, 892-912. [CrossRef]

13. Kraus, M.; Liimets, T.; Cappa, C.E.; Cidale, L.S.; Nickeler, D.H.; Duronea, N.U.; Arias, M.L.; Gunawan, D.S.; Oksala, M.E.; Fernandes, M.B.; et al. Resolving the Circumstellar Environment of the Galactic B[e] Supergiant Star MWC 137 from Large to Small Scales. Astron. J. 2017, 154, 186, [CrossRef]

14. Kraus, M.; Liimets, T.; Moiseev, A.; Sánchez Arias, J.P.; Nickeler, D.H.; Cidale, L.S.; Jones, D. Resolving the Circumstellar Environment of the Galactic B[e] Supergiant Star MWC 137.II. Nebular Kinematics and Stellar Variability. Astron. J. 2021, 162, 150, [CrossRef]

15. Afanasiev, V.L.; Moiseev, A.V. Scorpio on the $6 \mathrm{~m}$ Telescope: Current State and Perspectives for Spectroscopy of Galactic and Extragalactic Objects. Balt. Astron. 2011, 20, 363-370. [CrossRef]

16. Moiseev, A.V. Scanning Fabry-Perot Interferometer of the 6-m SAO RAS Telescope. Astrophys. Bull. 2021, 76, 316-339. [CrossRef]

17. Labrie, K.; Anderson, K.; Cárdenes, R.; Simpson, C.; Turner, J.E.H. DRAGONS—Data Reduction for Astronomy from Gemini Observatory North and South. In Astronomical Data Analysis Software and Systems XXVII; Teuben, P.J., Pound, M.W., Thomas, B.A., Warner, E.M., Eds.; ASP Conference Series; Astronomical Society of the Pacific: College Park, MD, USA, 2019.

18. Cochetti, Y.R.; Kraus, M.; Arias, M.L.; Cidale, L.S.; Eenmäe, T.; Liimets, T.; Torres, A.F.; Djupvik, A.A. Near-infrared Characterization of Four Massive Stars in Transition Phases. Astron. J. 2020, 160, 166, [CrossRef]

19. Miroshnichenko, A.S.; Fremat, Y.; Houziaux, L.; Andrillat, Y.; Chentsov, E.L.; Klochkova, V.G. High resolution spectroscopy of the galactic candidate LBV MWC 314. Astron. Astrophys. Suppl. 1998, 131, 469-478. [CrossRef]

20. Wisniewski, J.P.; Babler, B.L.; Bjorkman, K.S.; Kurchakov, A.V.; Meade, M.R.; Miroshnichenko, A.S. The Asymmetrical Wind of the Candidate Luminous Blue Variable MWC 314. Publ. Astron. Soc. Pac. 2006, 118, 820-827. [CrossRef]

21. Frasca, A.; Miroshnichenko, A.S.; Rossi, C.; Friedjung, M.; Marilli, E.; Muratorio, G.; Busà, I. Interpreting the spectral behavior of MWC 314. Astron. Astrophys. 2016, 585, A60, [CrossRef]

22. Lobel, A.; Groh, J.H.; Martayan, C.; Frémat, Y.; Torres Dozinel, K.; Raskin, G.; Van Winckel, H.; Prins, S.; Pessemier, W.; Waelkens, C.; et al. Modelling the asymmetric wind of the luminous blue variable binary MWC 314. Astron. Astrophys. 2013, 559, A16, [CrossRef]

23. Richardson, N.D.; Moffat, A.F.J.; Maltais-Tariant, R.; Pablo, H.; Gies, D.R.; Saio, H.; St-Louis, N.; Schaefer, G.; Miroshnichenko, A.S.; Farrington, C.; et al. Spectroscopy, MOST photometry, and interferometry of MWC 314: Is it an LBV or an interacting binary? Mon. Not. R. Astron. Soc. 2016, 455, 244-257. [CrossRef]

24. Reed, D.S.; Balick, B.; Hajian, A.R.; Klayton, T.L.; Giovanardi, S.; Casertano, S.; Panagia, N.; Terzian, Y. Hubble Space Telescope Measurements of the Expansion of NGC 6543: Parallax Distance and Nebular Evolution. Astron. J. 1999, 118, $2430-2441$. [CrossRef]

25. Liimets, T.; Corradi, R.L.M.; Jones, D.; Verro, K.; Santander-García, M.; Kolka, I.; Sidonio, M.; Kankare, E.; Kankare, J.; Pursimo, T.; et al. New insights into the outflows from R Aquarii. Astron. Astrophys. 2018, 612, A118, [CrossRef]

26. Gaia Collaboration. VizieR Online Data Catalog: Gaia DR2 (Gaia Collaboration, 2018). VizieR Online Data Catalog. 2018. I/345. Available online: https: / / ui.adsabs.harvard.edu/abs/2018yCat.1345....0G/abstract (accessed on 31 January 2022).

27. Bode, M.F.; O'Brien, T.J.; Simpson, M. Echoes of an Explosive Past: Solving the Mystery of the First Superluminal Source. Astrophys. J. 2004, 600, L63-L66. [CrossRef]

28. Arias, M.L.; Cidale, L.S.; Kraus, M.; Torres, A.F.; Aidelman, Y.; Zorec, J.; Granada, A. Near-infrared Spectra of a Sample of Galactic Unclassified B[e] Stars. Publ. Astron. Soc. Pac. 2018, 130, 114201. [CrossRef]

29. Mehner, A.; de Wit, W.J.; Groh, J.H.; Oudmaijer, R.D.; Baade, D.; Rivinius, T.; Selman, F.; Boffin, H.M.J.; Martayan, C. VLT/MUSE discovers a jet from the evolved B[e] star MWC 137. Astron. Astrophys. 2016, 585, A81, [CrossRef]

30. Bally, J.; Chia, Z.H. A highly collimated jet from the Red Square nebula MWC 922. Mon. Not. R. Astron. Soc. 2019, 484, 4529-4539. [CrossRef]

31. Antoniucci, S.; Podio, L.; Nisini, B.; Bacciotti, F.; Lagadec, E.; Sissa, E.; La Camera, A.; Giannini, T.; Schmid, H.M.; Gratton, R.; et al. Sub-0.1" optical imaging of the Z CMa jets with SPHERE/ZIMPOL. Astron. Astrophys. 2016, 593, L13, [CrossRef] 